\title{
The Importance of Anatomy and Dissection in the Human Corpse
}

\author{
Antonio Bernardes* \\ Associate Professor, University of Coimbra, Portugal
}

*Corresponding author: Antonio Bernardes, Associate Professor, University of Coimbra, Rua Antonio Jardim, Urbanizacao Quinta do Cedro, lote 1, 3000-036 Coimbra-Portugal, Tel: 00351919695799, Email: antoniojbernardes@gmail.com

Submission: 酱 October 7, 2017; Published: 眥 October 13, 2017

\section{Editorial}

Medical sciences have been enriched by new disciplines whose knowledge is imposed on future physicians. Therefore, it is necessary to make successive updates to the pre-graduate curriculum, the latter which are required to contemplate additions of modern subjects, while fundamental disciplines such as Anatomy have seen their hours reduced in some schools in a worrying way. Students are faced with the difficult reality of being forced to learn in a minimum period of time the most basic essential information to the understanding of other subjects and good medical practice.

At first Anatomy was almost nothing more than man's curiosity about his constitution. From the result of dissections, so often misunderstood and even punished socially and/or even judicially, and from the detailed observations was born the "pure" Descriptive Anatomy, which was taught for decades in a rather exhausting way. Subsequently, the learning of Morphology gained an indispensable status to the understanding the organic functioning that it described in detail, which contributed to the advent of Physiology. Anatomy grew in importance, was revered and respected. In the last decades, however, even with the worldwide recognition of its value, Anatomy lived through difficult days. Some comment that Anatomy has lost the leadership function, or that was important but now has no meaning, or that all Anatomy has already been discovered. We disagree completely with this defeatist pessimism because Anatomy is not an exhausted Science. It is very alive, its usefulness being eternal and infinite. How often do we use Anatomy or anatomical language in our daily activities without our understanding? It renews itself constantly, adapts itself, being called to the limelight whenever technical innovations of diagnosis and therapy appear. In addition, we are obliged to accept the importance of Anatomy in the interpretation of images captured by imaging techniques (ultrasound, tomography, magnetic resonance imaging), in celioscopic surgery, robotics, transplantation, and even in the production of prostheses.

Unfortunately, we have witnessed in several countries a decrease in the number of "anatomical vocations" for several reasons, but mostly because of clinical disciplines that are more attractive. It all starts on the benches of the School where some teachers do not want or cannot captivate interest because they often teach a pure Anatomy without indicating its benefit to medical practice. On the other hand, university career legislation in many countries does not facilitate and can even hinder career progression, by making it elitist rather than encouraging it, contrary to what happens in the hospital career. In addition, governments offer clinicians more and better economic conditions.

The deeper knowledge of diseases and technological evolution put to the service of Medicine and Surgery and the recent diagnostic and therapeutic possibilities obligate us today to teach Morphology by privileging its clinical applications. It must provide the indispensable bases for the interpretation of the ordinary and the pathological, and guide the surgeon with excellence: it is the Surgical anatomy. This applied, practical, or even clinic Anatomy has rapidly gained adherents all over the world, a reality well evidenced by the creation of specialized journals and the formation of scientific societies of clinical morphology.

Anatomy is a fundamental pillar of Medical Sciences, although there are many limitations and difficulties. One of the most important of the latter is undoubtedly the dissection in the corpse. Unfortunately, there is little pre- and post-graduate dissection in many schools, especially because they have few donors, although this is the technique that characterizes the teaching of anatomy, which gives it identity and utility. The theoretical teaching based on books, videos or computers can never replace the dissection that offers educational, intellectual, and emotional experience. Dissection is the student's first opportunity to contact the reality of the human body. It is an essential method of learning the anatomical structures and to understand the topography of the various regions. It also allows the student to feel the texture of the tissues and organs and to verify that the variation of normal is very frequent, more: that the normal in Anatomy is the variation. That is why a significant percentage of poor clinical practice is attributed to the lack of knowledge of anatomical variations. The dissection also serves to help the student to face agony and death in the future.

The difficulty in obtaining corpses for teaching and research has been compensated and overcome by the dissection of natural 
pieces that are later submitted to conservation techniques. These pieces include: lower limbs amputated by chronic ischemia, upper limbs amputated by trauma, cervical, thoracic and abdominal viscera collected during autopsies, etc. Its pedagogical value is inestimable and superior to that of plastic parts or even of computer programs. Following this perspective, we have carried out and fomented in our Faculty the dissection in human pieces and the respective preservation by means of techniques of parafinization, dehydration, corrosion of vascular casts, or simply freezing them or soaking them in formaldehyde.

Most students and physicians especially surgeons recognize the need for dissection to improve their training and manual dexterity and train new techniques on the cadaver before practicing them on the patient. This practical knowledge of the anatomical constitution will surely help them optimize and solve many diagnostic and therapeutic problems. 